\title{
Histórico familiar de câncer gástrico em pacientes dispépticos indicados à triagem endoscópica
}

Family history of gastric cancer in dyspeptic patients referred for endoscopic screening Antecedentes familiares de cáncer gástrico en pacientes dispépticos derivados a triaje endoscópico

Maria Carolina Pereira Rodrigues ${ }^{1}$ (c) https://orcid.org/0000-0003-0941-4851

Victor Pereira Lima ${ }^{1}$ ib https://orcid. org/0000-0002-5883-1267)

Flavia Ferreira Monari ${ }^{1}$ io https://orcid.org/0000-0001-6702-2982,

Roberta de Araújo e Silva ${ }^{1}$ iD https:/orcid.org/0000-0003-2133-0677.

Liana Mara Rocha Teles ${ }^{2}$ Io https://orcid.org/0000-0002-8124-5231

Eveline Pinheiro Beserra ${ }^{2}$ io https:/orcid.org/0000-0002-0631-8461

Maria Alzete de Lima ${ }^{3}$ io nttps://orididorg/0000-0002-0288-1329

Maria Aparecida Alves de Oliveira Serra' ${ }^{1}$ ib hittps://orcid.org/0000-0003-0952-9560

Como citar:

Rodrigues MC, Pereira VL, Monari FF, Silva RA, Teles LM, Beserra EP, et al. Histórico familiar de câncer gástrico em pacientes dispépticos indicados à triagem endoscópica. Acta Paul Enferm. 2021;34:AAPE001985.

DOI

http://dx.doi.org/10.37689/actaape/2021A0001985

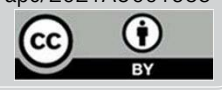

Dispepsia; Endoscopia; Gastropatias; Neoplasias gastrointestinais

Keywords

Dyspepsia; Endoscopy; Gastrointestinal neoplasms; Stomach diseases

Descriptores

Dispepsia; Endoscopía; Gastropatías; Neoplasias gastrointestinal

Submetido 27 de Julho de 2020

Aceito

8 de Março de 2021

Autor correspondente

Maria Aparecida Alves de Oliveira Serra Email: cidinhaenfaufc@yahoo.com.br

\section{Resumo}

Objetivo: Identificar o histórico familiar de primeiro grau de câncer gástrico em pacientes com sintomas dispépticos atendidos em um serviço público de endoscopia.

Métodos: Estudo transversal, realizado com pacientes dispépticos que tinham indicação para realizar o exame de endoscopia digestiva alta. A associação entre o histórico familiar de câncer gástrico e os resultados do exame endoscópico foi verificada por meio dos testes de Qui-quadrado ou Fisher, e medida seu efeito por meio da razão de chance e intervalo de confiança em analises uni e multivariadas. Utilizou-se regressão logística na análise dos dados.

Resultados: Observou-se que dos 751 pacientes dispépticos investigados, 44 (5,9\%) possuíam histórico familiar de câncer gástrico, destes a maioria era do sexo feminino $(70,5 \%)$, com idade maior ou igual a 45 anos (56,8\%). Os pacientes com histórico familiar de câncer gástrico tinham maiores chances de não apresentarem diagnostico endoscópico de úlcera péptica $(p=0,05 ; R C=2,33 ; I C=0,99-5,48)$. Além de maiores chances de alterações na mucosa gástrica $(p=0,05 ; R C=1,06 ; I C=1,04-1,08)$ e infecção pela Helicobacter pylori $(\mathrm{p}=0,04 ; \mathrm{RC}=1,79 ; \mathrm{IC}=0,94-3,39)$ mesmo após ajustes nas análises.

Conclusão: A alteração endoscópica da mucosa gástrica e a infecção pela Helicobacter pylori em pacientes com sintomas dispépticos, mostraram associação independente com o histórico familiar de câncer gástrico. Diante disso, faz-se necessário a elaboração de protocolos de assistência à saúde para melhor investigação e vigilância dos familiares de câncer gástrico, bem como ações de educação em saúde para orientar os pacientes a respeito do rastreio e prevenção do câncer gástrico.

\section{Abstract}

Objective: To identify first-degree relative history of gastric cancer in patients with dyspeptic symptoms receiving care at a public endoscopy service.

Methods: A cross-sectional study, performed with dyspeptic patients referred for an upper gastrointestinal endoscopy. The association between the family history of gastric cancer and the findings of the endoscopic examination was verified using the Chi-square or Fisher tests, and its effect was shown using odds ratio and confidence interval in univariate and multivariate analyses. Logistic regression was used to analyze the data.

Results: Among the 751 dyspeptic patients enrolled, 44 (5.9\%) had a family history of gastric cancer, mostly females $(70.5 \%)$ aged 45 years or older (56.8\%). Patients with a family history of gastric cancer were more likely to have no endoscopic diagnosis of peptic ulcer ( $\mathrm{p}=0.05 ; \mathrm{OR}=2.33 ; \mathrm{Cl}=0.99-5.48)$. In addition, higher chances of gastric mucosal changes $(p=0.05 ; R C=1.06 ; C l=1.04-1.08)$ and Helicobacter pylori infection $(p=0.04 ; \mathrm{RC}=1.79 ; \mathrm{Cl}=0.94-3.39)$ were found, even after adjusting the analyses.

${ }^{1}$ Universidade Federal do Maranhão, Imperatriz, MA, Brasil.

2Universidade Federal do Ceará, Fortaleza, CE, Brasil.

${ }^{3}$ Universidade Federal do Rio Grande do Norte, Natal, RN, Brasil.

Conflitos de interesse: nada a declarar. 
Conclusion: The endoscopic gastric mucosal changes and Helicobacter pylori infection in patients with dyspeptic symptoms showed an independent association with family history of gastric cancer. Therefore, it is necessary to develop health care protocols for better investigation and surveillance of gastric cancer relatives, as well as health education actions to guide patients regarding screening and prevention of gastric cancer.

\section{Resumen}

Objetivo: Identificar los antecedentes familiares de primer grado de cáncer gástrico en pacientes con síntomas dispépticos atendidos en un servicio público de endoscopía.

Métodos: Estudio transversal llevado a cabo con pacientes dispépticos que habían sido derivados a realizar un estudio de endoscopía digestiva alta. La relación entre los antecedentes familiares de cáncer gástrico y los resultados del estudio endoscópico fue verificada mediante la prueba $\chi^{2}$ de Pearson 0 de Fisher, y su efecto fue medido a través de la razón de momios y del intervalo de confianza en análisis uni y multivariados. Se utilizó la regresión logística en el análisis de los datos.

Resultados: Se observó que de los 751 pacientes dispépticos investigados, 44 (5,9\%) tenían antecedentes familiares de cáncer gástrico, de los cuales la mayoría era de sexo femenino (70,5\%), de 45 años o más (56,8 \%). Los pacientes con antecedentes familiares de cáncer gástrico tenían mayores chances de no presentar diagnóstico endoscópico de úlcera péptica $(\mathrm{p}=0,05 ; \mathrm{RC}=2,33 ; \mathrm{IC}=0,99-5,48)$. Además de mayores probabilidades de alteraciones en la mucosa gástrica $(p=0,05 ; R C=1,06 ; \mid C=1,04-1,08)$ e infección por Helicobacter pylori $(p=0,04 ; R C=1,79 ; I C=0,94-3,39)$, inclusive después de ajustes en los análisis.

Conclusión: La alteración endoscópica de la mucosa gástrica y la infección por Helicobacter pylori en pacientes con síntomas dispépticos mostraron relación independiente con los antecedentes familiares de cáncer gástrico. Ante este escenario, es necesaria la elaboración de protocolos de atención a la salud para una mejor investigación y observación de los familiares de cáncer gástrico, así como también acciones de educación en salud para orientar a los pacientes sobre la detección y prevención del cáncer gástrico.

\section{Introdução}

O câncer gástrico (CG) é a sexta neoplasia mais comum em todo o mundo e a terceira causa de morte relacionada ao câncer. ${ }^{(1)}$ Acredita-se que devido a intervençóes de saúde apropriadas, a incidência de CG está diminuindo nos países desenvolvidos, porém ainda permanece com elevadas taxas de morbimortalidade nos países em desenvolvimento. ${ }^{(2)}$

Múltiplos fatores estão envolvidos no aumento do risco de desenvolver GC como, baixas condições socioeconômicas, idade avançada, hábitos alimentares, etilismo, tabagismo, histórico familiar de câncer, infecçóes por vírus Epstein-Barr e Helicobacter pylori, além das lesóes pré-malignas do estômago. ${ }^{(3,4)}$ Devido à natureza multifatorial do CG, a sua prevenção está sujeita à identificação precisa dos fatores de risco, das causas subjacentes desta doença e manejo adequado desses fatores. ${ }^{(5)}$

A infecção por $H$. pylori e o histórico familiar de câncer gástrico têm sido relatados como fatores de risco importantes para o desenvolvimento de CG em todo o mundo. ${ }^{(6,7)}$ A erradicação da $H$. pylori foi definida como estratégia para prevenir o CG segundo publicação da Organização Mundial de Saúde (OMS) em 2014. ${ }^{(8)}$ Estudos têm demostrado resultados consistentes que a erradicação da $H$. pylori reduz o risco de CG, dependendo da gravidade e extensão dos danos na mucosa gástrica no momen- to da erradicação, e recomendam o tratamento de todos os indivíduos infectados. ${ }^{(9-11)}$

Membros da família dos pacientes com CG têm maiores taxas de infecção por $H$. pylori do que as pessoas na população geral e as alteraçóes histológicas pré-cancerosas do estômago são mais graves nesse grupo. ${ }^{(12,13)}$ Pacientes com CG e seus parentes compartilham fatores de risco, incluindo características genéticas e exposição a $H$. pylori no meio ambiente. Essa bactéria pode ser facilmente transmitida de pessoa para pessoa, visto que pode ser encontrada nas fezes, saliva e placa dentária, favorecendo a concentração da infecção entre familiares e pessoas que convivem no mesmo ambiente. ${ }^{(14,15)}$

Embora o histórico familiar seja um fator de risco reconhecido para o CG, à base molecular da agregação familiar e a transmissão intrafamiliar da H.pylori não são claras. $\mathrm{O}$ câncer gástrico familiar geralmente indica apenas um histórico familiar positivo, enquanto o câncer gástrico hereditário sugere alterações em genes específicos. ${ }^{(16)}$ Apesar das recomendaçôes atuais de rastrear e erradicar a H.pylori entre os membros familiares de pacientes com CG, as estratégias de acompanhamento, triagem e intervalo de vigilância neste grupo de alto risco, não foram bem estabelecidas pelas diretrizes mundiais. ${ }^{(15,17)}$

A identificação de indivíduos de alto risco é importante para a vigilância e prevenção do CG. $\mathrm{O}$ adequado diagnóstico e tratamento das lesóes da mucosa 
gástrica em estágios iniciais podem reduzir a mortalidade relacionada ao CG, além de contribuir para tratamentos eficazes, com menores custos e aumento da sobrevida. ${ }^{(18)}$ As diretrizes atuais recomendam a triagem endoscópica oportuna dos pacientes com sintomas dispépticos, para detectar doenças orgânicas que causam os sintomas do paciente, principalmente para descartar malignidades gastrointestinais superiores. ${ }^{(6)}$

Portando, investigar e conhecer o histórico familiar de câncer gástrico em pacientes com sintomas dispépticos são importantes para traçar estratégias de prevenção e controle do CG. Além de, conhecer as populaçóes mais susceptíveis, contribuindo para elaboração de protocolos de vigilância, tratamento e detecção precoce do CG. Diante disso, o presente estudo tem o objetivo de identificar o histórico familiar de primeiro grau de câncer gástrico em pacientes com sintomas dispépticos atendidos em um serviço público de endoscopia.

\section{Métodos}

Trata-se de estudo transversal que foi realizado em um serviço público de endoscopia, situado no nordeste do Brasil, com pacientes que apresentavam sintomas dispépticos e tinham indicação para realizar o exame de endoscopia digestiva alta (EDA). O cálculo da amostra foi realizado por uma fórmula para população infinita. Adotou-se uma prevalência de $50 \%$ por proporcionar um tamanho amostral máximo, nível de confiança de 95\% $(Z \alpha=1,96)$ e erro amostral de 5\%. Para melhor representatividade da amostra, o tamanho da mesma foi acrescido em 10\% ( $\mathrm{n}=751$ pacientes com sintomas dispépticos).

A seleção dos participantes foi realizada aleatoriamente, obedecendo aos critérios de elegibilidade estabelecidos. Os critérios de inclusão foram: pacientes com idade mínima de 18 anos de ambos os sexos, com indicação para realizar o exame de endoscopia digestiva alta. Os critérios de exclusão foram: uso de antibióticos ou antissecretores gástricos nas últimas duas semanas que antecedem o exame de EDA, grávidas ou em lactação, condiçóes associadas a distúrbios da fisiologia gástrica como, vagotomia, cirurgia prévia de ressecção gástrica, estenose pilórica.

A coleta de dados foi realizada no período de Outubro de 2015 a fevereiro de 2018, na sala de espera do serviço de endoscopia. O recrutamento dos pacientes foi realizado na sala de espera do exame de EDA, após os esclarecimentos sobre os objetivos e a metodologia da pesquisa. Os que concordaram, assinaram o Termo de Consentimento Livre e Esclarecido (TCLE) e participaram da entrevista. $\mathrm{O}$ instrumento utilizado para coleta de dados foi um formulário contendo dados de identificação, características socioeconômicas, clinicas e histórico familiar de primeiro grau de câncer gástrico.

Os diagnósticos endoscópicos foram consultados no prontuário do paciente. A detecção da $H$. pylori foi realizada por meio do teste rápido de uréase, durante o exame endoscopia digestiva alta. $\mathrm{O}$ teste rápido da urease baseia-se na produção de urease pela bactéria para efetuação do diagnóstico indireto da presença da $H$. pylori. ${ }^{(19)}$

Os dados foram analisados com o software SPSS for Windows, versão 22.0 (SPSS Inc., Chicago, IL). Primeiramente foi aplicado o teste de Kolmogorov-Smirnov para avaliar a normalidade das variáveis quantitativas, que foram apresentadas por meio de estatística descritiva (media e desvio padrão). As correlaçóes entre as variáveis do estudo foram avaliadas pelos testes de Quiquadrado ou Fisher. O nível de significância foi estabelecido em p-value $\leq 0,05$. Elegeram-se como variável dependente: o histórico familiar de câncer gástrico e variáveis independentes: os resultados do exame de endoscopia digestiva alta e a presença da infecção pelo Helicobacter pylori. Para determinar a relação entre a variável dependente e o conjunto simultâneo de variáveis independentes a análise de regressão logística foi utilizada.

Foram respeitadas as normas nacionais e internacionais que regem a ética em pesquisa envolvendo seres humanos. Este estudo foi aprovado pelo Comitê de Ética em Pesquisa da instituição responsável pelo estudo com parecer $n^{\circ}$ 1.304.308. 


\section{Resultados}

Foram analisados 751 pacientes com sintomas dispépticos atendidos em um serviço público de endoscopia, com predomínio do sexo feminino $(68,3 \%)$, a idade variou entre 18 e 91 anos, com média de idade de 43,43 (desvio padrão de 16,42), 66,7\% eram casados, $67,2 \%$ tinham renda familiar mensal de mais de um salário mínimo ( $\mathrm{R} \$ 1851,41)$, $50,3 \%$ estudaram por um tempo superior a oito anos, $82 \%$ se declararam náo tabagistas, $67,5 \%$ não etilistas e $94,1 \%$ não possui histórico de câncer gástrico na família.

Nesse estudo, os pacientes com sintomas dispépticos que apresentaram idade superior a 45 anos tiveram maiores chances de serem casados $(\mathrm{p}<0,0001$; $\mathrm{RC}=2,87$; IC:2,07 - 3,98), estudarem por um tempo igual ou inferior a oito anos $(\mathrm{p}<0,0001 ; \mathrm{RC}=$ 2,93; IC: $2,40-3,58)$ e tabagistas $(\mathrm{p}<0,0001$; $\mathrm{RC}=$ $1,58$; IC: $1,35-1,85)$. Os pacientes com idade inferior ou igual a 45 anos possuíam maiores chances de serem etilistas $(p=0,02 ; R C=1,44$; IC: $1,05-1,97)$ e apresentarem alteraçóes endoscópicas da mucosa gástrica $(\mathrm{p}=0,01 ; \mathrm{RC}=2,31 ; \mathrm{IC}: 1,20-4,45)$, além de menores chances de apresentarem diagnostico de esofagite ( $p=0,004 ; \mathrm{RC}=0,61$; IC: $0,44-0,85)$. Não houve associação entre idade e a presença de histórico familiar de câncer gástrico, como pode ser observada na tabela 1 .

Observou-se que 44 (5,9\%) pacientes possuíam histórico familiar de câncer gástrico, sendo a maioria do sexo feminino $(70,5 \%)$, com idade maior ou igual a 45 anos $(56,8 \%)$, renda superior a um salário mínimo $(68,2 \%)$, escolaridade acima de oito anos $(52,3 \%)$, não tabagistas $(81,8 \%)$ e não etilistas $(59,1 \%)$. Esses fatores socioeconômicos não estavam associados à histórico familiar de câncer gástrico, como mostra a tabela 2.

Os pacientes com sintomas dispépticos que relataram histórico familiar de câncer gástrico tinham maior chance de alteraçâo da mucosa gástrica e todos apresentaram resultados alterados no exame de esofagastroduodenoscopia $(\mathrm{p}=0,05 ; \mathrm{RC}=1,06$; IC: $1,04-1,08)$, além de maior chance de estarem infectados pela H.pylori ( $\mathrm{p}=0,04 ; \mathrm{RC}=1,79$; IC: 0,94 $-3,39)$. Observou-se que os pacientes dispépticos
Tabela 1. Distribuição das características socioeconômicas e clínicas dos pacientes com sintomas dispépticos atendidos em um serviço público de endoscopia, segundo idade

\begin{tabular}{|c|c|c|c|c|c|}
\hline \multirow[b]{2}{*}{ Variáveis } & \multicolumn{2}{|c|}{ Idade } & \multirow[b]{2}{*}{$p$-value } & \multirow[b]{2}{*}{$\mathrm{RC}$} & \multirow[b]{2}{*}{$95 \% \mathrm{IC}$} \\
\hline & $\begin{array}{c}<=45 \\
\mathrm{n}=413 \\
\mathrm{n}(\%)\end{array}$ & $\begin{array}{c}>45 \\
\mathrm{n}=338 \\
\mathrm{n}(\%)\end{array}$ & & & \\
\hline \multicolumn{6}{|l|}{ Sexo } \\
\hline Feminino & $278(67,4)$ & $235(69,6)$ & 0,51 & 1,10 & $0,81-1,51$ \\
\hline \multicolumn{6}{|l|}{ Estado Civil } \\
\hline Casado & $234(56,7)$ & $267(78,9)$ & $<0,0001^{*}$ & 2,87 & $2,07-3,98$ \\
\hline \multicolumn{6}{|l|}{ Renda } \\
\hline 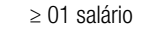 & $284(68,8)$ & $221(65,3)$ & 0,32 & 0,85 & $0,63-1,16$ \\
\hline \multicolumn{6}{|l|}{ Escolaridade } \\
\hline$\leq 8$ anos & $125(30,2)$ & $253(74,8)$ & $<0,0001^{*}$ & 2,93 & $2,40-3,58$ \\
\hline \multicolumn{6}{|l|}{ Tabagista } \\
\hline Sim & $48(11,6)$ & $87(25,7)$ & $<0,0001^{*}$ & 1,58 & $1,35-1,85$ \\
\hline \multicolumn{6}{|l|}{ Etilista } \\
\hline Sim & $149(36,1)$ & $95(28,1)$ & $0,02^{*}$ & 1,44 & $1,05-1,97$ \\
\hline \multicolumn{6}{|c|}{$\begin{array}{l}\text { Histórico familiar de } \\
\text { câncer gástrico }\end{array}$} \\
\hline Sim & $19(4,6)$ & $25(7,3)$ & 0,10 & 0,60 & $0,32-1,11$ \\
\hline \multicolumn{6}{|c|}{$\begin{array}{l}\text { Alteração na Mucosa } \\
\text { Gástrica }\end{array}$} \\
\hline Sim & $35(8,4)$ & $13(3,8)$ & $0,01^{*}$ & 2,31 & $1,20-4,45$ \\
\hline \multicolumn{6}{|c|}{ Infecção pela H. pylori } \\
\hline Sim & $220(53,2)$ & $176(52,1)$ & 0,74 & 1,04 & $0,78-1,39$ \\
\hline \multicolumn{6}{|l|}{ Gastrite } \\
\hline Sim & $323(78,2)$ & $270(79,8)$ & 0,57 & 0,90 & $0,63-1,28$ \\
\hline \multicolumn{6}{|l|}{ Úlcera Peptica } \\
\hline Sim & $27(6,5)$ & $33(9,7)$ & 0,10 & 0,64 & $0,38-1,09$ \\
\hline \multicolumn{6}{|l|}{ Esofagite } \\
\hline Sim & $90(21,7)$ & $105(31,1)$ & $0,004^{*}$ & 0,61 & $2,06-2,41$ \\
\hline
\end{tabular}

Tabela 2. Associação dos fatores socioeconômicos com o histórico familiar de câncer gástrico em pacientes com sintomas dispépticos

\begin{tabular}{|c|c|c|c|c|c|}
\hline \multirow[b]{2}{*}{ Variáveis } & & \multicolumn{2}{|c|}{$\begin{array}{l}\text { Histórico familiar de } \\
\text { câncer gástrico }\end{array}$} & \multirow[b]{2}{*}{ Total } & \multirow[b]{2}{*}{$p$-value } \\
\hline & & $\begin{array}{c}\text { Sim } \\
(n=44) \\
f(\%)\end{array}$ & $\begin{array}{c}\text { Não } \\
(n=707) \\
f(\%)\end{array}$ & & \\
\hline \multirow[t]{2}{*}{ Sexo } & Masculino & $13(29,5)$ & $225(31,8)$ & 238 & \multirow[t]{2}{*}{0,448} \\
\hline & Feminino & $31(70,5)$ & $482(68,2)$ & 513 & \\
\hline \multirow[t]{2}{*}{ Idade } & $\leq 45$ anos & $19(43,2)$ & $394(55,7)$ & 413 & \multirow[t]{2}{*}{0,072} \\
\hline & $\geq 45$ anos & $25(56,8)$ & $313(44,3)$ & 338 & \\
\hline \multirow[t]{2}{*}{ Renda } & $\leq 01$ salário & $14(31,8)$ & $232(32,8)$ & 246 & \multirow[t]{2}{*}{0,518} \\
\hline & $\geq 01$ salário & $30(68,2)$ & $475(67,2)$ & 505 & \\
\hline \multirow[t]{2}{*}{ Estado Civil } & Solteiro & $12(27,3)$ & $238(33,7)$ & 250 & \multirow[t]{2}{*}{0,242} \\
\hline & Outros & $32(72,7)$ & $469(66,3)$ & 501 & \\
\hline \multirow[t]{2}{*}{ Escolaridade } & $\leq 08$ anos & $21(47,7)$ & $357(50,5)$ & 378 & \multirow[t]{2}{*}{0,420} \\
\hline & $\geq 08$ anos & $23(52,3)$ & $350(49,5)$ & 373 & \\
\hline \multirow[t]{2}{*}{ Fumante } & Sim & $8(18,2)$ & $127(18,0)$ & 135 & \multirow[t]{2}{*}{0,551} \\
\hline & Não & $36(81,8)$ & $580(82,0)$ & 616 & \\
\hline \multirow[t]{2}{*}{ Etilista } & Sim & $18(40,9)$ & $226(32,0)$ & 244 & \multirow[t]{2}{*}{0,144} \\
\hline & Não & $26(59,1)$ & $481(68,0)$ & 507 & \\
\hline
\end{tabular}

$\mathrm{f}=$ frequência 
Tabela 3. Associação dos resultados do exame endoscópico com a presença de histórico familiar de câncer gástrico em pacientes com sintomas dispépticos

\begin{tabular}{|c|c|c|c|c|c|c|c|}
\hline \multicolumn{8}{|c|}{$\begin{array}{l}\text { Histórico familiar de câncer } \\
\text { gástrico }\end{array}$} \\
\hline \multicolumn{2}{|l|}{ Resultados endoscópicos } & $\begin{array}{c}\text { Sim } \\
(n=44) \\
f(\%)\end{array}$ & $\begin{array}{c}\text { Não } \\
(\mathrm{n}=707) \\
\mathrm{f}(\%)\end{array}$ & Total & $p$-value & $\mathrm{RC}$ & IC $95 \%$ \\
\hline \multirow[t]{2}{*}{ Alteração na Mucosa Gástrica } & Sim & $44(100,0)$ & $659(93,2)$ & 703 & $0,05^{\star}$ & 1,06 & $1,04-1,08$ \\
\hline & Não & $00(0,0)$ & $48(6,8)$ & 48 & & & \\
\hline \multirow[t]{2}{*}{ Infecção pelo H. pylori } & Sim & $29(65,9)$ & $367(51,9)$ & 396 & $0,04^{*}$ & 1,79 & $0,94-3,39$ \\
\hline & Não & $15(34,1)$ & $340(48,1)$ & 355 & & & \\
\hline \multirow[t]{2}{*}{ Gastrite } & Sim & $38(86,4)$ & $555(78,5)$ & 593 & 0,14 & 1,73 & $0,72-4,18$ \\
\hline & Não & $06(13,6)$ & $152(21,5)$ & 158 & & & \\
\hline \multirow[t]{2}{*}{ Gastrite de Antro } & Sim & $33(75,0)$ & $478(67,6)$ & 511 & 0,19 & 1,43 & $0,71-2,89$ \\
\hline & Não & $11(25,0)$ & $229(32,4)$ & 240 & & & \\
\hline \multirow[t]{2}{*}{ Gastrite de Corpo } & Sim & $2(4,5)$ & $37(5,2)$ & 39 & 0,59 & 0,86 & $0,20-3,70$ \\
\hline & Não & $42(95,5)$ & $670(94,8)$ & 712 & & & \\
\hline \multirow[t]{2}{*}{ Úlcera Péptica } & Sim & $7(15,9)$ & $53(7,5)$ & 60 & $0,05^{\star}$ & 2,33 & $0,99-5,48$ \\
\hline & Não & $37(84,1)$ & $654(92,5)$ & 691 & & & \\
\hline \multirow[t]{2}{*}{ Esofagite } & Sim & $10(22,7)$ & $185(26,2)$ & 195 & 0,38 & 0,83 & $0,40-1,71$ \\
\hline & Não & $34(77,3)$ & $522(73,8)$ & 556 & & & \\
\hline
\end{tabular}

*p $\leq 0.05 ; \mathrm{F}=$ frequência; $\mathrm{RC}=$ Razão de chance; IC 95\% = Intervalo de confiança de $95 \%$

com histórico familiar de câncer gástrico tiveram maior chance de não apresentar o diagnostico de úlcera péptica ( $\mathrm{p}=0,05 ; \mathrm{RC}=2,33$; IC: $0,99-5,48)$, como observado na tabela 3 .

Após análise multivariada, os pacientes com histórico familiar de câncer gástrico permaneceram no modelo com maiores chances de apresentarem alteração endoscópica da mucosa gástrica $(\mathrm{p}<0,0001$; $\mathrm{RC}=2,34 ; \mathrm{IC}=1,10-5,51)$ e infecção pela H.pylori $(\mathrm{p}=0,05 ; \mathrm{RC}=1,89 ; \mathrm{IC}=0,84-3,18)$.

\section{Discussão}

O presente estudo evidenciou que o histórico familiar de câncer gástrico estava presente em 5,9\% dos pacientes e a maioria era do sexo feminino, com idade igual ou superior a 45 anos. A influência do histórico familiar de câncer gástrico na suscetibilidade ao CG pode diferir de acordo com o sexo. Estudo de revisão mostrou que indivíduos com histórico familiar de câncer gástrico de primeiro grau do sexo feminino foram associados a um risco aumentado de desenvolver CG. ${ }^{(20)}$

O limite de idade no risco de desenvolver CG pode diferir entre as regióes de alto e baixo risco, aproximadamente 45 anos e 50 anos de idade, respetivamente. ${ }^{(6)}$ Em indivíduos com histórico familiar o limite é de 50 anos de idade ou possuir cerca de 10 anos de diferença mais novo que o parente de primeiro grau no diagnóstico. ${ }^{(7)}$

Evidenciou-se no presente estudo que os pacientes dispépticos com idade superior a 45 anos tiveram maiores chances de serem casados, com menor escolaridade e tabagistas. A idade avançada, ${ }^{(3)}$ baixa escolaridade $^{(21)}$ e o tabagismo ${ }^{(22)}$ são fatores de risco relevantes para o desenvolvimento do CG. Assim, sugere-se o planejamento de ações em saúde para cessação do consumo de tabaco, por meio de assistência integral, adequadas a idade e escolaridade dos pacientes, a fim de prevenir o aparecimento do CG.

$\mathrm{O}$ estudo atual mostrou que os pacientes dispépticos com idade igual ou menor que 45 anos tinham maiores chances de serem etilistas e apresentarem alteraçóes endoscópicas da mucosa gástrica. Estudos sugeriram que o consumo excessivo de álcool está associado a um aumento do risco de CG. ${ }^{(23,24)}$ Diante disso, açôes em saúde que oriente os pacientes dispépticos com alteraçóes na mucosa gástrica a reduzirem o consumo de álcool, podem ser favoráveis na prevenção do CG.

Uma hipótese bem estabelecida para o desenvolvimento do CG resulta da progressão de lesôes precursoras, na qual a inflamação crônica da mucosa gástrica (gastrite crônica) progride, por meio de condiçóes pré-neoplásicas como gastrite atrófica, metaplasia intestinal e displasia, para a neoplasia gástrica. ${ }^{(25)}$ 
No presente estudo, a alteração endoscópica mais frequente nos pacientes com histórico familiar de câncer gástrico foi à gastrite, além destes apresentarem maiores chances de alteraçóes endoscópicas da mucosa gástrica, mesmo após ajustes nas analises. Esses dados reforçam a necessidade de vigilância endoscópica nesse grupo de pacientes, como forma de prevenção do aparecimento das lesóes pré-cancerosa no estômago.

Os pacientes dispépticos com histórico familiar de câncer gástrico investigados neste estudo tinham maiores chances de estarem infectados pela H.pylori, nas análises uni e multivariadas. Esses dados sugerem a transmissão intrafamiliar da bactéria e reforçam a necessidade de erradicação da H.pylori e confirmação da eliminação da infecção, com a finalidade de controlar a transmissão e reduzir as complicações a longo prazo.

A forma como a $H$. pylori é transmitida ainda é incerta, sugerindo que a principal via seja a transmissão interpessoal, embora a transmissão ambiental por meio da água e alimentos contaminados permaneça possível. ${ }^{(26)}$ Estudos mostram que o ambiente familiar parece relevante na transmissáo pessoa a pessoa, principalmente a transmissão de mãe para filho, visto que a maioria das infecçóes por $H$. pylori ocorre na infância e resultam no aparecimento de doenças gástricas na idade adulta. ${ }^{(17,27)}$

Encontrou-se que os pacientes com histórico familiar de câncer gástrico tiveram maior chance de não apresentarem o diagnostico de úlcera péptica. Esses dados estáo de acordo com estudo de Nishizawa et $\mathrm{al}^{\left({ }^{(28)}\right.}$ onde se evidenciou que o histórico familiar em primeiro grau de câncer gástrico, ausência de úlceras pépticas e idade avançada foram fatores de risco independentes para atrofia gástrica em pacientes infectados por $H$. pylori.

Durante o desenvolvimento desta pesquisa, deparou-se com limitaçôes como, possuir amostra oriunda de um único serviço de endoscopia. Por ser um estudo transversal não foi possível o acompanhamento dos pacientes do estudo. A coleta das informaçôes acerca do histórico familiar de câncer gástrico foi baseada nas recordaçóes dos pacientes, o que pode propiciar um viés de memória.
Portanto, reconhecer os fatores de risco relevantes para o desenvolvimento do câncer gástrico entre os pacientes com sintomas dispépticos, contribui para expandir e aperfeiçoar o papel da enfermagem em saúde pública na educação, prática, pesquisa e esforços na elaboração de politicas e protocolos de vigilância, tratamento e prevenção no controle do câncer gástrico, especialmente para grupos de pacientes com maior risco de desenvolver a doença, como os familiares de câncer gástrico.

\section{Conclusão}

O estudo evidenciou que os pacientes com sintomas dispépticos com histórico familiar de câncer gástrico tinham maiores chances de apresentaram alteraçóes na mucosa gástrica e infecção pela $H$. pylori, fatores de risco relevantes para o desenvolvimento do câncer gástrico. Diante dos achados do presente estudo, faz-se necessário a elaboração de protocolos de assistência à saúde para melhor investigação e vigilância dos familiares de câncer gástrico, bem como açóes de educação em saúde para orientar os pacientes a respeito do rastreio e prevenção do câncer gástrico.

\section{Agradecimentos}

Os autores agradecem à Fundação de Amparo à Pesquisa e ao Desenvolvimento Cientifico e Tecnológico do Maranhão (FAPEMA) por seu apoio no desenvolvimento da pesquisa. Processo UNIVERSAL-00420/15. Ao Conselho Nacional de Ciência e Tecnologia (CNPq) por seu apoio em bolsas de iniciação cientifica.

\section{Colaborações}

Rodrigues MCP, Lima VP, Monari FF, Silva RA, Teles LMR, Beserra EP, Lima MA e Serra MAAO contribuíram com a concepção do estudo, análise e interpretação dos dados, redação do artigo, revisão crítica relevante do conteúdo intelectual e aprovação da versão final a ser publicada. 


\section{Referências}

1. Bray F, Ferlay J, Soerjomataram I, Siegel RL, Torre LA, Jemal A. Global cancer statistics 2018: GLOBOCAN estimates of incidence and mortality worldwide for 36 cancers in 185 countries. CA Cancer J Clin. 2018;68(6):394-424. Erratum in: CA Cancer J Clin. 2020;70(4):313.

2. Thrift AP, El-Serag HB. Burden of Gastric Cancer. Clin Gastroenterol Hepatol. 2020;18(3):534-42. Review.

3. Machlowska J, Baj J, Sitarz M, Maciejewski R, Sitarz R. Gastric Cancer: Epidemiology, Risk Factors, Classification, Genomic Characteristics and Treatment Strategies. Int J Mol Sci. 2020;21(11):4012. Review.

4. Yoon H, Kim N. Diagnosis and management of high risk group for gastric cancer. Gut Liver. 2015;9(1):5-17. Review.

5. Yusefi AR, Bagheri Lankarani K, Bastani P, Radinmanesh M, Kavosi Z. Risk Factors for Gastric Cancer: A Systematic Review. Asian Pac J Cancer Prev. 2018;19(3):591-603. Review.

6. Quach DT, Hiyama T, Gotoda T. Identifying high-risk individuals for gastric cancer surveillance from western and eastern perspectives: Lessons to learn and possibility to develop an integrated approach for daily practice. World J Gastroenterol. 2019;25(27):3546-62. Review.

7. Kim GH, Liang PS, Bang SJ, Hwang JH. Screening and surveillance for gastric cancer in the United States: Is it needed? Gastrointest Endosc. 2016;84(1):18-28. Review.

8. International Agency for Research on Cancer (IARC). IARC Working Group Report. Helicobacter pylori Eradication as a Strategy for Preventing Gastric Cancer. vol.8. France: IARC; 2014 [cited 2021 Feb 20]. Available from: https://publications.iarc.fr/Book-And-ReportSeries/larc-Working-Group-Reports/-Em-Helicobacter-Pylori-EmEradication-As-A-Strategy-For-Preventing-Gastric-Cancer-2014

9. Khan MY, Aslam A, Mihali AB, Shabbir Rawala M, Dirweesh A, Khan S, et al. Effectiveness of Helicobacter pylori eradication in preventing metachronous gastric cancer and preneoplastic lesions. A systematic review and metaanalysis. Eur J Gastroenterol Hepatol. 2020;32(6):686-94.

10. Sugano K. Effect of Helicobacter pylori eradication on the incidence of gastric cancer: a systematic review and meta-analysis. Gastric Cancer. 2019;22(3):435-45.

11. Lee YC, Chiang TH, Chou CK, Tu YK, Liao WC, Wu MS, et al. Association Between Helicobacter pylori Eradication and Gastric Cancer Incidence: a Systematic Review and Meta-analysis. Gastroenterology. 2016;150(5):1113-24.e5. Review.

12. Choi YJ, Kim N, Jang W, Seo B, Oh S, Shin CM, et al. Familial Clustering of Gastric Cancer: A Retrospective Study Based on the Number of FirstDegree Relatives. Medicine (Baltimore). 2016;95(20):e3606.

13. Youn Nam S, Park BJ, Nam JH, Ryu KH, Kook MC, Kim J, et al. Association of current Helicobacter pylori infection and metabolic factors with gastric cancer in 35,519 subjects: a cross-sectional study. United European Gastroenterol J. 2019;7(2):287-96.
14. Yaghoobi M, McNabb-Baltar J, Bijarchi R, Hunt RH. What is the quantitative risk of gastric cancer in the first-degree relatives of patients? A meta-analysis. World J Gastroenterol. 2017;23(13):243542.

15. Choi IJ, Kim CG, Lee JY, Kim YI, Kook MC, Park B, et al. Family history of gastric cancer and helicobacter pylori treatment. $\mathrm{N}$ Engl J Med. 2020;382(5):427-36.

16. Boland $\mathrm{CR}$, Yurgelun MB. Historical perspective on familial gastric cancer. Cell Mol Gastroenterol Hepatol. 2017;3(2):192-200. Review.

17. Ding SZ. Global whole family based-Helicobacter pylori eradication strategy to prevent its related diseases and gastric cancer. World $\mathrm{J}$ Gastroenterol. 2020;26(10):995-1004. Review.

18. de Souza Giusti AC, de Oliveira Salvador PT, Dos Santos J, Meira KC, Camacho AR, Guimarães RM, et al. Trends and predictions for gastric cancer mortality in Brazil. World J Gastroenterol. 2016;22(28):652738

19. Uotani T, Graham DY. Diagnosis of Helicobacter pylori using the rapid urease test. Ann Transl Med. 2015;3(1):9. Review.

20. Choi YJ, Kim N. Gastric cancer and family history. Korean J Intern Med. 2016;31(6):1042-53. Review.

21. Rota M, Alicandro G, Pelucchi C, Bonzi R, Bertuccio P, Hu J, et al. Education and gastric cancer risk-an individual participant data metaanalysis in the StoP project consortium. Int J Cancer. 2020;146(3):671 81. Erratum in: Int J Cancer. 2020;146(11):E6.

22. Poorolajal J, Moradi L, Mohammadi Y, Cheraghi Z, Gohari-Ensaf F. Risk factors for stomach cancer: a systematic review and meta-analysis. Epidemiol Health. 2020;42:e2020004.

23. Tramacere I, Negri E, Pelucchi C, Bagnardi V, Rota M, Scotti L, et al. A meta-analysis on alcohol drinking and gastric cancer risk. Ann Oncol. 2012;23(1):28-36.

24. Rota M, Pelucchi C, Bertuccio P, Matsuo K, Zhang ZF, Ito H, et al. Alcohol consumption and gastric cancer risk-a pooled analysis within the StoP project consortium. Int J Cancer. 2017;141(10):1950-62. Erratum in: Int J Cancer. 2018;143(8):E10.

25. Li S, Chung DC, Mullen JT. Screening high-risk populations for esophageal and gastric cancer. J Surg Oncol. 2019;120(5):831-46.

26. Mladenova I, Durazzo M. Transmission of Helicobacter pylori. Minerva Gastroenterol Dietol. 2018;64(3):251-4. Review.

27. Urita Y, Watanabe T, Kawagoe N, Takemoto I, Tanaka H, Kijima S, et al. Role of infected grandmothers in transmission of Helicobacter pylori to children in a Japanese rural town. J Paediatr Child Health. 2013;49(5):394-8

28. Nishizawa T, Suzuki H, Sakitani K, Yamashita H, Yoshida S, Hata K, et al. Family history is an independent risk factor for the progression of gastric atrophy among patients with Helicobacter pylori infection. United European Gastroenterol J. 2017;5(1):32-6. 